\title{
Business Cycles, Bank Credit and Crises
}

\author{
Monika Bucher Diemo Dietrich Achim Hauck
}

March 6, 2013

\begin{abstract}
This paper investigates how business cycle volatility affects internal and external funding sources of banks. It argues that excessive credit growth, credit cycles, and bank failures are phenomena related to distinct patterns of banks' financing options over the cycle. This perspective reconciles rational behavior with some implications of Minsky's financial instability hypothesis.
\end{abstract}

Keywords bank lending, banking crisis, credit crunch, cyclicality

JEL Classification G01, G21, E32

\section{Introduction}

There is a close link between business cycles, bank credit, and banking crises. According to Jordà at al. (2011), production growth, investment spending and credit growth over the last 140 years were the smaller the more credit-intense the preceding boom had been. In addition, financial crises were often accompanied by deep and lasting recessions.

A possible explanation follows the financial instability hypothesis of Minsky (1986), according to which a period of prolonged prosperity may induce speculative euphoria and excess borrowing which push the economy on the brink. This view became popular during the recent world financial crisis and challenged the consensus macroeconomic models based on rational behavior of agents (Davidson, 2008). In response, financial frictions became more central in these models (Gertler and Kiyotaki, 2010). However, they still have difficulties in explaining excessive credit growth or discontinuous phenomena such as bank failures.

We present a model that focuses on the role of real volatility as a potential cause for the dynamics and stability of credit supply. The model reconciles rational behavior with some implications of the financial instability hypothesis. We argue that bank stability and the dynamics of credit are related to banks' internal and external funding problems, for which 
an important driver is the business cycle. In a downturn, internal funding sources dry up as existing loans generate only small cash flows and may even cause a debt overhang. External funding is hampered as the funding liquidity of new loans is low in a weak environment with a risky outlook. Bank stability and credit growth then depend on the relative importance of these effects. In a downturn with a gloomy outlook, banks cannot fully refinance credit by borrowing against their future earnings. To prevent such liquidity shortages, banks may fuel excessive credit growth in good times to improve internal funding in later bad times. However, if internal funding in downturns is insensitive to previous credit expansion, banks will instead gamble for resurrection implying a failure should the recovery hold off. Finally, if loans become too toxic in a downturn, banks will fail outright.

\section{Set up}

Consider a bank that lives for three dates. At $t=0$ and $t=1$ it raises funds from depositors, invests $a_{t}$ in a risk-free asset with zero net return, and grants loans $l_{t}$. At $t=1$, the economy either is in benign conditions ("good" state $g$, probability $p_{1}$ ) or experiences a downturn ("bad" state $b$, probability $1-p_{1}$ ). In the good state, loans granted at $t=0$ earn a high return $v_{g}$ and loans granted at $t=1$ yield either $r_{h, g}$ (with probability $p_{2, g}$ ) or $r_{l, g}<r_{h, g}$ at $t=2 .{ }^{1}$ If the bad state occurs at $t=1$, early loans yield only $v_{b}<v_{g}$ and late loans yield $r_{h, b}<r_{l, g}$ if the economy recovers (with probability $p_{2, b}$ ) and $r_{l, b}<1$ otherwise. We assume $p_{1} v_{g}>1$ and $p_{2, s} r_{h, s}>1$. Given constant means $\mu_{1}:=p_{1} v_{g}+\left(1-p_{1}\right) v_{b}$ and $\mu_{2, s}:=p_{2, s} r_{h, s}+\left(1-p_{2, s}\right) r_{l, s}$, loan earnings read

$$
\begin{array}{ll}
v_{g}=\mu_{1}+\left(1-p_{1}\right) \Delta_{1}, & r_{h, s}=\mu_{2, s}+\left(1-p_{2, s}\right) \Delta_{2, s}, \\
v_{b}=\mu_{1}-p_{1} \Delta_{1}, & r_{l, s}=\mu_{2, s}-p_{2, s} \Delta_{2, s} .
\end{array}
$$

with mean preserving spreads $\Delta_{1}:=v_{g}-v_{b}$ and $\Delta_{2, s}:=r_{h, s}-r_{l, s}$ to measure risks associated with lending at $t=0$ and $t=1$ respectively. $^{2}$

\footnotetext{
${ }^{1}$ Unless otherwise indicated all returns are per unit.

${ }^{2}$ The returns thus exhibit persistent and mean reverting shocks, which is common in macromodels (cf. Aghion et al., 2010).
} 
Originating loans is costly for the bank. Costs $c\left(l_{t}\right)$ are non-pecuniary, increasing and convex with $c(0)=c^{\prime}(0)=0$. This captures the idea that loans differ only in their complexity and a bank adds the least complex loans first to its portfolio. Depositors inelastically provide funds to the bank at a zero interest rate. In return, the bank promises at $t-1$ to repay at $t$ the face value of deposits $d_{t}$. If it fails to keep the promise, a bank run completely destroys all values. All agents are risk neutral and have no time preference.

\section{Analysis}

The bank can choose between three modes $m_{t}$ of operation at $t=0$ and $t=1$. In the safe mode $\mathcal{S}$, deposits are always repaid at the next date. The corresponding expected profits are $\phi_{0}^{\mathcal{S}}\left(l_{0}\right)=\left(\mu_{1}-1\right) l_{0}-c\left(l_{0}\right)$ and $\phi_{1, s}^{\mathcal{S}}\left(l_{1, s}\right)=\left(\mu_{2, s}-1\right) l_{1, s}-c\left(l_{1, s}\right)$, respectively. In the risky mode $\mathcal{R}$, deposits are repaid only if earnings are large and the expected profits are only $\phi_{0}^{\mathcal{R}}\left(l_{0}\right)=\left(p_{1} v_{g}-1\right) l_{0}-c\left(l_{0}\right)$ and $\phi_{1, s}^{\mathcal{R}}\left(l_{1, s}\right)=\left(p_{2, s} r_{h, s}-1\right) l_{1, s}-c\left(l_{1, s}\right)$. In the failure mode $\mathcal{F}$, the bank declares bankruptcy. Note that first best loan volumes $l_{0}^{\mathrm{fb}}$ and $l_{1, s}^{\mathrm{fb}}$ are given by $\phi_{0}^{\mathcal{S} \prime}\left(l_{0}^{\mathrm{fb}}\right)=0$ and $\phi_{1, s}^{\mathcal{S} \prime}\left(l_{1, s}^{\mathrm{fb}}\right)=0$.

We proceed by backward induction. Let $\Omega_{1, s}:=v_{s} l_{0}+a_{0}-d_{1} \lessgtr 0$ be the cash flow at $t=1$ in state $s=g, b$ and $q_{2, s}$ the probability of a bank run at $t=2$. Unless the bank operates in the failure mode as from $t=1$, its optimization problem reads

$$
\begin{aligned}
& \max _{l_{1, s}, a_{1, s}, d_{2, s} \in \mathbb{R}^{+}} \pi_{1, s}=E {\left[\max \left\{r_{j, s} l_{1, s}+a_{1, s}-d_{2, s}, 0\right\}\right]-c\left(l_{1, s}\right) } \\
& \text { s.t. } l_{1, s}+a_{1, s}= \Omega_{1, s}+\left(1-q_{2, s}\right) d_{2, s}, \\
& q_{2, s}= \begin{cases}0 & \text { if } m_{1, s}=\mathcal{S}: d_{2, s} \leq r_{l, s} l_{1, s}+a_{1, s}, \\
1-p_{2, s} & \text { if } m_{1, s}=\mathcal{R}: d_{2, s} \in\left(r_{l, s} l_{1, s}+a_{1, s}, r_{h, s} l_{1, s}+a_{1, s}\right],\end{cases}
\end{aligned}
$$

with $j=h, l$. The budget constraint (4) states that loans $l_{1, s}$ and assets $a_{1, s}$ are refinanced internally by the cash flow $\Omega_{1, s}$ and externally by fresh deposits of face value $d_{2, s}$. According to (5) a run occurs at $t=2$ if the face value of deposits $d_{2, s}$ exceeds total earnings $r_{j, s} l_{1, s}+a_{1, s}$. Since all values are destroyed in a run, depositors invest $\left(1-q_{2, s}\right) d_{2, s}$ at $t=1$. If there is no interior solution that yields a positive expected profit for the bank, it will fail at $t=1$. 
This decision problem is best understood when the comparative advantages and disadvantages of the safe and the risky mode are spelled out. The advantage of the safe mode at $t=1$ is that the bank collects all earnings irrespective of the economy's state. The disadvantage is that lending is subject to a financial restriction in the bad state. This restriction results from the upper limit on the face value of deposits $\left(d_{2, b} \leq r_{l, b} l_{1, b}+a_{1, b}\right)$ which in conjunction with (4) and (5) requires

$$
\Omega_{1, b}+\left(r_{l, b}-1\right) l_{1, b} \geq 0 .
$$

Since $r_{l, b}<1$, the bank cannot fully refinance loans at $t=1$ externally in the bad state. Their funding liquidity is negative. Lending is then feasible only if the bank commands additional internal funds, i.e. $\Omega_{1, b}>0$. As these funds are limited, the loan volume $l_{1, b}$ is bounded above by $l_{1, b}^{\max }:=\frac{\Omega_{1, b}}{1-r_{l, b}}=\frac{\Omega_{1, b}}{p_{2, b}\left(\Delta_{2, b}-\widetilde{\Delta}_{2, b}\right)}$ with $\widetilde{\Delta}_{2, b}:=\frac{\mu_{2, b}-1}{p_{2, b}} .3$ The advantage of the risky over the safe mode is that no upper limit on lending exists as depositors can be promised up to $r_{h, s} l_{1, s}+a_{1, s}$. Although the bank will keep this promise only with probability $p_{2, s}$, it suffices to refinance any new loan since $p_{2, s} r_{h, s}>1$. The disadvantage is that with probability $1-p_{2, s}$ a run destroys all values. Lending is thus less profitable.

Let $\pi_{1, s}^{*}$ denote the bank's expected profits at $t=1$ given the optimal loans size $l_{1, s}^{*}$ and mode of operation $m_{1, s}^{*}$ at this date in state $s=g, b$. As long as the bank does not fail at $t=0$, its decision problem reads

$$
\begin{aligned}
\max _{l_{0}, a_{0}, d_{1} \in \mathbb{R}^{+}} \pi_{0} & =p_{1} \pi_{1, g}^{*}+\left(1-p_{1}\right) \pi_{1, b}^{*}-c\left(l_{0}\right) \\
\text { s.t. } l_{0}+a_{0} & =\left(1-q_{1}\right) d_{1}, \\
q_{1} & =\left\{\begin{array}{ll}
0 & \text { if } m_{0}=\mathcal{S}: m_{1, g}^{*} \neq \mathcal{F} \text { and } m_{1, b}^{*} \neq \mathcal{F} \\
1-p_{1} & \text { if } m_{0}=\mathcal{R}: m_{1, g}^{*} \neq \mathcal{F} \quad \text { and } m_{1, b}^{*}=\mathcal{F}
\end{array},\right.
\end{aligned}
$$

with $q_{1}$ being the probability of a run at $t=1$. A subgame-perfect strategy maximizes the bank's expected profits $\pi_{0}$ taking into account its future decisions, the budget constraint (8), and the probability (9) with which it fails to repay the face value of deposits at $t=1$.

\footnotetext{
${ }^{3}$ Such an upper bound is not an issue in the good state, in which the funding liquidity of loans is positive as $r_{l, g}>1$.
} 
For a bank that opts for the safe mode at $t=0$, internal and external funds are abundantly available if the economy turns out to be in benign conditions at $t=1$ as both, the realized returns $v_{g}$ on old loans and the minimum returns $r_{l, g}$ on new loans are greater than the opportunity costs of funds. If the economy slows down, the financial position of the bank at $t=1$ crucially depends on how much it collects from the loans granted at $t=0$ and how much it owes to depositors. The budget constraint (8) implies $\Omega_{1, b}=\left(v_{b}-1\right) l_{0}=p_{1}\left(\widetilde{\Delta}_{1}-\Delta_{1}\right) l_{0}$ with $\widetilde{\Delta}_{1}:=\frac{\mu_{1}-1}{p_{1}}$. Hence, the bank cannot proceed with the safe mode if $\Delta_{1}>\widetilde{\Delta}_{1}$ as it then suffers from a debt overhang $\left(\Omega_{1, b}<0\right)$, which is the higher the more loans have been granted at $t=0$. If $\Delta_{1}<\widetilde{\Delta}_{1}$, internal funds ease the financial constraint in a downturn $\left(\Omega_{1, b}>0\right)$. Moreover, funds available for new loans are the greater, the more loans were granted at $t=0$. This follows from rewriting the upper limit on loans $l_{1, b}^{\max }$ to

$$
l_{1, b}^{\max }=\frac{p_{1}\left(\widetilde{\Delta}_{1}-\Delta_{1}\right)}{p_{2, b}\left(\Delta_{2, b}-\widetilde{\Delta}_{2, b}\right)} l_{0}=: \psi l_{0}
$$

where $\psi$ measures how many loans could be additionally refinanced in a downturn if one more unit was granted at $t=0$; it is smaller for higher risks $\Delta_{1}$ or $\Delta_{2, b}$.

Against this background, the bank's decision is ultimately a choice between five strategies which can be put in a strict order. Strategy A is to always operate in the safe mode and lend according to the first best. This strategy is available as long as the upper limit on loans at $t=1$ in a downturn is not binding, $l_{1, b}^{\max } \geq l_{1, b}^{\mathrm{fb}}$, i.e. if risks are small or if $l_{0}^{\mathrm{fb}}$ is large relative to $l_{1, b}^{\mathrm{fb}}$.

When the restriction $l_{1, b}^{\max }$ binds, the next best alternative is strategy $\mathrm{B}$, which is to maintain the safe mode and to ease this restriction by increasing loans at $t=0$ above the first best level in order to mitigate funding problems in a downturn at $t=1$. The bank thus deviates from the first best in a way that one may interpret as excessive credit growth at $t=0$ turning into a credit crunch at $t=1$. Note that higher risks not only imply a tighter financial constraint in a downturn by impairing internal funds and the funding liquidity of new loans. They also imply a weaker impact of $l_{0}$ on this constraint. Hence, the inefficiencies associated with a deviation from the first best in both periods become more of a burden for higher risks. 
Above a certain risk level, the bank thus pursues strategy $\mathrm{C}$ which is to always grant loans according to the first best in good times and to switch to the risky mode in a downturn, implying a bank run if the recovery from the downturn holds off. If risks associated with $l_{0}$ are even larger, lending according to the first best at $t=0$ would already be associated with a substantial debt overhang in a downturn. Anticipating that the bank would find it optimal to default on its debt in this situation, depositors are not willing to refinance so many loans at $t=0$. Accordingly, strategy $\mathrm{D}$ is to signal credibility to depositors by not raising too much debt at $t=0$, implying that loans are bounded above by some $l_{0}^{\max }$ already at this date. As for risks associated with $l_{1, b}$, they do not come with such disincentives. Quite the contrary, since a bank that already speculates on the upside can only benefit from a further wedge between the returns in the recovery and the non-recovery state, a larger recovery risk allows the bank to cover a higher debt overhang without suffering from a loss of confidence. An observer could easily interpret this pattern as banks making little provisions for bad times in the presence of significant risks throughout the cycle and responding to emerging financial difficulties mainly by taking on a fragile capital structure.

Finally, for very large risks $\Delta_{1}$ attached to loans in the first period, strategy E is adopted which is to pursue the risky mode already at $t=0$. Lending at $t=0$ will be suppressed as loan earnings can only be collected when the economy does not slip into a downturn. This case may be representative for poor and least developed economies that experience substantial difficulties in growing out of their financial problems.

Formally, the bank's optimal decisions $m_{0}^{*}, l_{0}^{*}$ at $t=0$ and $m_{1, b}^{*}, l_{1, b}^{*}$ in the downturn at $t=1$ satisfy $^{4}$

$$
\begin{aligned}
& m_{0}^{*}=\mathcal{S}, \quad l_{0}^{*}=l_{0}^{\mathrm{fb}}, \quad m_{1, b}^{*}=\mathcal{S}, \quad l_{1, b}^{*}=l_{1, b}^{\mathrm{fb}} \quad \text { if } \quad \Delta_{1} \leq \Delta_{A} \\
& m_{0}^{*}=\mathcal{S}, \quad l_{0}^{*}=l_{0}^{\mathcal{S}}>l_{0}^{\mathrm{fb}}, \quad m_{1, b}^{*}=\mathcal{S}, \quad l_{1, b}^{*}=\psi l_{0}^{\mathcal{S}}<l_{1, b}^{\mathrm{fb}} \quad \text { if } \quad \Delta_{1} \in\left(\Delta_{A}, \Delta_{B}\right] \\
& m_{0}^{*}=\mathcal{S}, \quad l_{0}^{*}=l_{0}^{\mathrm{fb}}, \quad m_{1, b}^{*}=\mathcal{R}, \quad l_{1, b}^{*}=l_{1, b}^{\mathcal{R}}<l_{1, b}^{\mathrm{fb}} \quad \text { if } \quad \Delta_{1} \in\left(\Delta_{B}, \Delta_{C}\right] \\
& m_{0}^{*}=\mathcal{S}, \quad l_{0}^{*}=l_{0}^{\max }<l_{0}^{\mathrm{fb}}, \quad m_{1, b}^{*}=\mathcal{R}, \quad l_{1, b}^{*}=l_{1, b}^{\mathcal{R}}<l_{1, b}^{\mathrm{fb}} \quad \text { if } \quad \Delta_{1} \in\left(\Delta_{C}, \Delta_{D}\right] \\
& m_{0}^{*}=\mathcal{R}, \quad l_{0}^{*}=l_{0}^{\mathcal{R}}<l_{0}^{\mathrm{fb}}, \quad m_{1, b}^{*}=\mathcal{F}, \quad l_{1, b}^{*}=0 \quad \text { if } \quad \Delta_{1}>\Delta_{D} .
\end{aligned}
$$

\footnotetext{
${ }^{4}$ In the good state at $t=1$, the bank always chooses $m_{1, g}^{*}=\mathcal{S}$ and $l_{1, g}^{*}=l_{1, g}^{\mathrm{fb}}$.
} 
with

$$
\begin{array}{ll}
\Delta_{A}:=\widetilde{\Delta}_{1}-\frac{p_{2, b}\left(\Delta_{2, b}-\widetilde{\Delta}_{2, b}\right)}{p_{1}} \frac{l_{1, b}^{\mathrm{fb}}}{l_{0}^{\mathrm{fb}}}, & \Delta_{B}:\left(1-p_{1}\right)\left[\phi_{1, b}^{\mathcal{S}}\left(\psi l_{0}^{\mathcal{S}}\right)-\phi_{1, b}^{\mathcal{R}}\left(l_{1, b}^{\mathcal{R}}\right)\right]=\phi_{0}^{\mathcal{S}}\left(l_{0}^{\mathrm{fb}}\right)-\phi_{0}^{\mathcal{S}}\left(l_{0}^{\mathcal{S}}\right), \\
\Delta_{C}:=\widetilde{\Delta}_{1}+\frac{\phi_{1, b}^{\mathcal{R}}\left(l_{1, b}^{\mathcal{R}}\right)}{p_{1} l_{0}^{\mathrm{fb}}}, & \Delta_{D}:\left(1-p_{1}\right) \phi_{1, b}^{\mathcal{R}}\left(l_{1, b}^{\mathcal{R}}\right)=\phi_{0}^{\mathcal{R}}\left(l_{0}^{\mathcal{R}}\right)-\phi_{0}^{\mathcal{S}}\left(\frac{\phi_{1, b}^{\mathcal{R}}\left(l_{1, b}^{\mathcal{R}}\right)}{p_{1}\left(\Delta_{D}-\widetilde{\Delta}_{1}\right)}\right), \\
l_{0}^{\mathcal{R}}: \phi_{0}^{\mathcal{R} \prime}\left(l_{0}^{\mathcal{R}}\right)=0, & l_{0}^{\mathcal{S}}:\left(1-p_{1}\right) \psi \phi_{1, b}^{\mathcal{S} \prime}\left(\psi l_{0}^{\mathcal{S}}\right)+\phi_{0}^{\mathcal{S} \prime}\left(l_{0}^{\mathcal{S}}\right)=0, \\
l_{1, s}^{\mathcal{R}}: \phi_{1, s}^{\mathcal{R} \prime}\left(l_{1, s}^{\mathcal{R}}\right)=0, & l_{0}^{\max }:=\frac{\phi_{1, b}^{\mathcal{R}}\left(l_{1, b}^{\mathcal{R}}\right)}{p_{1}\left(\Delta_{1}-\widetilde{\Delta}_{1}\right)} .
\end{array}
$$

\section{Concluding remarks}

Our analysis abstracts from any repercussion from the banking sector to the business cycle. Although there is little doubt on these effects, turning them off not only sharpens the focus on the role of real volatility as a potential cause for the dynamics and stability of credit supply. It also confirms conventional wisdom that macroeconomic policy has a role to play for financial stability. In our model the outcome is constrained Pareto efficient. The performance of banks is thus improved only if the real economy is stabilized, for which a credible macro policy can make an important contribution.

\section{References}

Aghion, P., Angeletos, G.M., Banerjee, A., Manova, K., 2010. Volatility and Growth: Credit Constraints and the Composition of Investment. Journal of Monetary Economics 57, 246 265.

Davidson, P., 2008. Is the Current Financial Distress Caused by the Subprime Mortgage Crisis a Minsky Moment? Or Is it the Result of Attempting to Securitize Illiquid Noncommercial Mortgage Loans? Journal of Post Keynesian Economics 30, 669-676.

Gertler, M., Kiyotaki, N., 2010. Financial Intermediation and Credit Policy in Business Cycle Analysis, in: Friedman, B.M., Woodford, M. (Eds.), Handbook of Monetary Economics, volume 3. Elsevier, San Diego, pp. 547 - 599.

Jordà, Ò., Schularick, M., Taylor, A., 2011. When Credit Bites Back: Leverage, Business Cycles, and Crises. NBER Working Papers 17621. 
Minsky, H., 1986. Stabilizing an Unstable Economy: A Twentieth Century Fund Report. Yale University Press, New Haven. 


\section{Appendix}

We derive the optimal behavior of the bank in each period in three steps. First, we determine its behavior and expected payoff in the safe mode. Then, we proceed analogously for the risky mode. As a last step, we derive the actual behavior of the bank by comparing the respective payoffs with the payoff of failure. We apply backward induction.

\section{Second Period Optimum}

\section{Safe Mode}

For $m_{1, s}=\mathcal{S}$, insertion of $q_{2, s}=0$ into (4) yields $d_{2, s}=l_{1, s}+a_{1, s}-\Omega_{1, s}$. Insertion of this,

(1) and (2) into (3) and the restriction (5) on $d_{2, s}$ yields $\max _{l_{1, s}} \pi_{1, s}=\phi_{1, s}^{\mathcal{S}}\left(l_{1, s}\right)+\Omega_{1, s}$ s.t. $p_{2, s}\left(\Delta_{2, s}-\widetilde{\Delta}_{2, s}\right) l_{1, s} \leq \Omega_{1, s}$ with $\widetilde{\Delta}_{2, s}:=\frac{\mu_{2, s}-1}{p_{2, s}}$. Note that $\pi_{1, s}^{\prime}=\phi_{1, s}^{\mathcal{S} \prime}\left(l_{1, s}\right)=0$ for $l_{1, s}=l_{1, s}^{\mathrm{fb}}$, $\pi_{1, s}^{\prime \prime}<0$ and recall from the main text that $r_{l, g}>1>r_{l, b}$, which implies $\Delta_{2, g}<\widetilde{\Delta}_{2, g}$ and $\Delta_{2, b}>\widetilde{\Delta}_{2, b}$. Defining $l_{1, g}^{\min }:=-\frac{\Omega_{1, g}}{p_{2, g}\left(\widetilde{\Delta}_{2, g}-\Delta_{2, g}\right)}$ and $l_{1, b}^{\max }:=\frac{\Omega_{1, b}}{p_{2, b}\left(\Delta_{2, b}-\widetilde{\Delta}_{2, b}\right)}$ and denoting the (partial) optimum by the superscript $\mathcal{S} *$, we obtain

$$
\begin{array}{ll}
l_{1, g}^{\mathcal{S} *}=\max \left\{l_{1, g}^{\mathrm{fb}}, l_{1, g}^{\min }\right\}, & \pi_{1, g}^{\mathcal{S} *}=\phi_{1, g}^{\mathcal{S}}\left(\max \left\{l_{1, g}^{\mathrm{fb}}, l_{1, g}^{\min }\right\}\right)+\Omega_{1, g}, \\
l_{1, b}^{\mathcal{S} *}=\min \left\{l_{1, b}^{\mathrm{fb}}, l_{1, b}^{\max }\right\}, & \pi_{1, b}^{\mathcal{S} *}=\phi_{1, b}^{\mathcal{S}}\left(\min \left\{l_{1, b}^{\mathrm{fb}}, l_{1, b}^{\max }\right\}\right)+\Omega_{1, b}
\end{array}
$$

\section{Risky Mode}

For $m_{1, s}=\mathcal{R}$, insertion of $q_{2, s}=1-p_{2, s}$ into (4) yields $d_{2, s}=\frac{l_{1, s}+a_{1, s}-\Omega_{1, s}}{p_{2, s}}$. Insertion of this into (3) yields $\max _{l_{1, s}, a_{1, s}} \pi_{1, s}=\phi_{1, s}^{\mathcal{R}}\left(l_{1, s}\right)-\left(1-p_{2, s}\right) a_{1, s}+\Omega_{1, s}$. We ignore the restriction (5) on $d_{2, s}$ for the moment but show below that it is met when the bank adopts the risky mode. Since $a_{1, s}^{\mathcal{R} *}=0$, we obtain $\max _{l_{1, s}} \pi_{1, s}=\phi_{1, s}^{\mathcal{R}}\left(l_{1, s}\right)+\Omega_{1, s}$. Note that $\pi_{1, s}^{\prime}=\phi_{1, s}^{\mathcal{R} \prime}\left(l_{1, s}\right)=0$ for $l_{1, s}=l_{1, s}^{\mathcal{R}}<l_{1, s}^{\mathrm{fb}}$ where $l_{1, s}^{\mathcal{R}}: \phi_{1, s}^{\mathcal{R} \prime}\left(l_{1, s}^{\mathcal{R}}\right)=0$ and $\pi_{1, s}^{\prime \prime}<0$. We obtain

$$
l_{1, s}^{\mathcal{R} *}=l_{1, s}^{\mathcal{R}}, \quad \pi_{1, s}^{\mathcal{R} *}=\phi_{1, s}^{\mathcal{R}}\left(l_{1, s}^{\mathcal{R}}\right)+\Omega_{1, s}
$$

\section{Optimal Behavior}

We now compare $\pi_{1, s}^{\mathcal{S} *}$ and $\pi_{1, s}^{\mathcal{R} *}$ with the expected payoff $\pi_{1, s}^{\mathcal{F} *}=0$ of the failure mode. 
Good State If $\Omega_{1, g} \geq 0$, then (A.1) and (A.3) imply $\pi_{1, g}^{\mathcal{S} *}>\pi_{1, g}^{\mathcal{R} *}>\pi_{1, g}^{\mathcal{F} *}$. We can conclude

$$
m_{1, g}^{*}=\mathcal{S}, \quad l_{1, g}^{*}=l_{1, g}^{\mathrm{fb}}, \quad \pi_{1, g}^{*}=\phi_{1, g}^{\mathcal{S}}\left(l_{1, g}^{\mathrm{fb}}\right)+\Omega_{1, g} \quad \text { if } \quad \Omega_{1, g} \geq 0 .
$$

If $\Omega_{1, g}<0$, then (A.1) and (A.3) imply $\pi_{1, g}^{\mathcal{S} *}<\phi_{1, g}^{\mathcal{S}}\left(l_{1, g}^{\mathrm{fb}}\right)$ and $\pi_{1, g}^{\mathcal{R} *}<\phi_{1, g}^{\mathcal{S}}\left(l_{1, g}^{\mathrm{fb}}\right)$. We can conclude

$$
\pi_{1, g}^{*}<\phi_{1, g}^{\mathcal{S}}\left(l_{1, g}^{\mathrm{fb}}\right) \quad \text { if } \quad \Omega_{1, g}<0 .
$$

Bad State If $\Omega_{1, b} \geq 0$, then (A.2) and (A.3) imply $l_{1, b}^{\max } \geq 0, \pi_{1, b}^{\mathcal{S} *} \geq 0$ and $\pi_{1, b}^{\mathcal{R} *}>0$. We can conclude

$$
\begin{aligned}
& m_{1, b}^{*}=\mathcal{S}, l_{1, b}^{*}=l_{1, b}^{\mathrm{fb}}, \quad \pi_{1, b}^{*}=\phi_{1, b}^{\mathcal{S}}\left(l_{1, b}^{\mathrm{fb}}\right)+\Omega_{1, b} \text { if } l_{1, b}^{\max } \geq l_{1, b}^{\mathrm{fb}}, \\
& m_{1, b}^{*}=\mathcal{S}, l_{1, b}^{*}=l_{1, b}^{\max }, \pi_{1, b}^{*}=\phi_{1, b}^{\mathcal{S}}\left(l_{1, b}^{\max }\right)+\Omega_{1, b} \text { if } l_{1, b}^{\max } \in\left[0, l_{1, b}^{\mathrm{fb}}\right), \phi_{1, b}^{\mathcal{S}}\left(l_{1, b}^{\max }\right) \geq \phi_{1, b}^{\mathcal{R}}\left(l_{1, b}^{\mathcal{R}}\right), \\
& m_{1, b}^{*}=\mathcal{R}, l_{1, b}^{*}=l_{1, b}^{\mathcal{R}}, \quad \pi_{1, b}^{*}=\phi_{1, b}^{\mathcal{R}}\left(l_{1, b}^{\mathcal{R}}\right)+\Omega_{1, b} \text { if } l_{1, b}^{\max } \in\left[0, l_{1, b}^{\mathrm{fb}}\right), \phi_{1, b}^{\mathcal{S}}\left(l_{1, b}^{\max }\right)<\phi_{1, b}^{\mathcal{R}}\left(l_{1, b}^{\mathcal{R}}\right) .
\end{aligned}
$$

If $\Omega_{1, b}<0$, then (A.2) and (A.3) imply $l_{1, b}^{\max }<0, \pi_{1, b}^{\mathcal{S} *}<0$ and $\pi_{1, b}^{\mathcal{R} *} \lessgtr 0$. We can conclude

$$
\begin{array}{ll}
m_{1, b}^{*}=\mathcal{R}, l_{1, b}^{*}=l_{1, b}^{\mathcal{R}}, \pi_{1, b}^{*}=\phi_{1, b}^{\mathcal{R}}\left(l_{1, b}^{\mathcal{R}}\right)+\Omega_{1, b} & \text { if } l_{1, b}^{\max }<0, \Omega_{1, b} \in\left[-\phi_{1, b}^{\mathcal{R}}\left(l_{1, b}^{\mathcal{R}}\right), 0\right], \\
m_{1, b}^{*}=\mathcal{F}, l_{1, b}^{*}=0, \quad \pi_{1, b}^{*}=0 & \text { if } l_{1, b}^{\max }<0, \Omega_{1, b}<-\phi_{1, b}^{\mathcal{R}}\left(l_{1, b}^{\mathcal{R}}\right) .
\end{array}
$$

Since $m_{1, b}^{*}=\mathcal{R}$ only if $l_{1, b}^{\mathcal{R}}>l_{1, b}^{\max }$ and $\Omega_{1, b} \geq-\phi_{1, b}^{\mathcal{R}}\left(l_{1, b}^{\mathcal{R}}\right)$, the restriction (5) on $d_{2, s}$ will be met.

\section{First Period Optimum}

\section{Safe Mode}

For $m_{0}=\mathcal{S}$, insertion of $q_{1}=0$ into (8) yields $d_{1}=l_{0}+a_{0}$. Insertion of this, (1) and (2) into $\Omega_{1, s}:=v_{s} l_{0}+a_{0}-d_{1}$ for $s=g, b$ yields $\Omega_{1, g}=\left(\mu_{1}+\left(1-p_{1}\right) \Delta_{1}-1\right) l_{0} \geq 0$ and $\Omega_{1, b}=\left(\mu_{1}-p_{1} \Delta_{1}-1\right) l_{0} \lessgtr 0$ so that (A.4) implies $\pi_{1, g}^{*}=\phi_{1, g}^{\mathcal{S}}\left(l_{1, g}^{\mathrm{fb}}\right)+\Omega_{1, g}$ while (A.6) and (A.7) imply $\pi_{1, b}^{*}=\phi_{1, b}^{m_{1, b}^{*}}\left(l_{1, b}^{*}\right)+\Omega_{1, b}$ for $m_{1, b}^{*} \neq \mathcal{F}$. Insertion of $\Omega_{1, g}, \Omega_{1, b}, \pi_{1, g}^{*}$ and $\pi_{1, b}^{*}$ into (7) and the restriction $(9)$ on $m_{1, s}^{*}$ yields $\max _{l_{0}} \pi_{0}=p_{1} \phi_{1, g}^{\mathcal{S}}\left(l_{1, g}^{\mathrm{fb}}\right)+\left(1-p_{1}\right) \phi_{1, b}^{m_{1, b}^{*}}\left(l_{1, b}^{*}\right)+\phi_{0}^{\mathcal{S}}\left(l_{0}\right)$ 
s.t. $l_{0} \leq \frac{\phi_{1, b}^{\mathcal{R}}\left(l_{1, b}^{\mathcal{R}}\right)}{p_{1}\left(\Delta_{1}-\widetilde{\Delta}_{1}\right)}=: l_{0}^{\max }$ if $\Delta_{1}>\widetilde{\Delta}_{1}$. This implies

$$
\pi_{0}^{\prime}=\left(1-p_{1}\right) \phi_{1, b}^{m_{1, b}^{*}}\left(l_{1, b}^{*}\right) \frac{\partial l_{1, b}^{*}}{\partial l_{0}}+\phi_{0}^{\mathcal{S} \prime}\left(l_{0}\right)
$$

Note that $l_{1, b}^{\max }$ as defined in (A.2) can be redefined to $l_{1, b}^{\max }:=\psi l_{0}$ with $\psi:=\frac{p_{1}\left(\widetilde{\Delta}_{1}-\Delta_{1}\right)}{p_{2, b}\left(\Delta_{2, b}-\widetilde{\Delta}_{2, b}\right)}$ and consider the following two cases.

Case I Suppose that $\Delta_{1} \leq \widetilde{\Delta}_{1}$ and thus $\psi \geq 0$ and $l_{1, b}^{\max } \geq 0$.

- If $m_{1, b}^{*}=\mathcal{R}$, then (A.6) implies $\frac{\partial l_{1, b}^{*}}{\partial l_{0}}=0$. Insertion of this in (A.8) yields $\pi_{0}^{\prime}=\phi_{0}^{\mathcal{S} \prime}\left(l_{0}\right)=$ 0 for $l_{0}=l_{0}^{\mathrm{fb}}$ and $\pi_{0}^{\prime \prime}<0$. Denoting the (partial) optimum by the superscript $\mathcal{S} \mathcal{R} *$, we obtain

$$
l_{0}^{\mathcal{S R} *}=l_{0}^{\mathrm{fb}}, \quad \pi_{0}^{\mathcal{S R} *}=p_{1} \phi_{1, g}^{\mathcal{S}}\left(l_{1, g}^{\mathrm{fb}}\right)+\left(1-p_{1}\right) \phi_{1, b}^{\mathcal{R}}\left(l_{1, b}^{\mathcal{R}}\right)+\phi_{0}^{\mathcal{S}}\left(l_{0}^{\mathrm{fb}}\right) \quad \text { if } \quad \psi \geq 0 .
$$

- If $m_{1, b}^{*}=\mathcal{S}$, then (A.6) implies $\frac{\partial l_{1, b}^{*}}{\partial l_{0}}=\psi$ for $\psi l_{0} \in\left[0, l_{1, b}^{\mathrm{fb}}\right)$ and $\frac{\partial l_{1, b}^{*}}{\partial l_{0}}=0$ for $\psi l_{0} \geq l_{1, b}^{\mathrm{fb}}$. Insertion of this in (A.8) yields $\pi_{0}^{\prime}=\left(1-p_{1}\right) \psi \phi_{1, b}^{\mathcal{S \prime}}\left(\psi l_{0}\right)+\phi_{0}^{\mathcal{S} \prime}\left(l_{0}\right)$ for $\psi l_{0} \in\left[0, l_{1, b}^{\mathrm{fb}}\right)$ and $\pi_{0}^{\prime}=\phi_{0}^{\mathcal{S}}{ }^{\prime}\left(l_{0}\right)$ for $\psi l_{0} \geq l_{1, b}^{\mathrm{fb}}$. This has two implications. (a) If $\psi l_{0}^{\mathrm{fb}} \geq l_{1, b}^{\mathrm{fb}}$, then $\pi_{0}^{\prime}=0$ for $l_{0}=l_{0}^{\mathrm{fb}}$ and $\pi_{0}^{\prime \prime}<0$. (b) If $\psi l_{0}^{\mathrm{fb}}<l_{1, b}^{\mathrm{fb}}$, then $\pi_{0}^{\prime}=0$ for $l_{0}=l_{0}^{\mathcal{S}}$, where $l_{0}^{\mathcal{S}}:\left(1-p_{1}\right) \psi \phi_{1, b}^{\mathcal{S}}\left(\psi l_{0}^{\mathcal{S}}\right)+\phi_{0}^{\mathcal{S}}\left(l_{0}^{\mathcal{S}}\right)=0$ and $\pi_{0}^{\prime \prime}<0$. Accordingly, we have

$$
\begin{array}{ll}
l_{0}^{\mathcal{S S} *}=l_{0}^{\mathrm{fb}}, & \pi_{0}^{\mathcal{S S} *}=p_{1} \phi_{1, g}^{\mathcal{S}}\left(l_{1, g}^{\mathrm{fb}}\right)+\left(1-p_{1}\right) \phi_{1, b}^{\mathcal{S}}\left(l_{1, b}^{\mathrm{fb}}\right)+\phi_{0}^{\mathcal{S}}\left(l_{0}^{\mathrm{fb}}\right) \quad \text { if } \quad \psi l_{0}^{\mathrm{fb}} \geq l_{1, b}^{\mathrm{fb}}, \\
l_{0}^{\mathcal{S S} *}=l_{0}^{\mathcal{S}}, & \pi_{0}^{\mathcal{S} *}=p_{1} \phi_{1, g}^{\mathcal{S}}\left(l_{1, g}^{\mathrm{fb}}\right)+\left(1-p_{1}\right) \phi_{1, b}^{\mathcal{S}}\left(\psi l_{0}^{\mathcal{S}}\right)+\phi_{0}^{\mathcal{S}}\left(l_{0}^{\mathcal{S}}\right) \quad \text { if } \quad \psi l_{0}^{\mathrm{fb}} \in\left[0, l_{1, b}^{\mathrm{fb}}\right) .
\end{array}
$$

Note that if $\psi l_{0}^{\mathrm{fb}} \geq l_{1, b}^{\mathrm{fb}}$ and thus $\Delta_{1} \leq \widetilde{\Delta}_{1}-\frac{p_{2, b}\left(\Delta_{2, b}-\widetilde{\Delta}_{2, b}\right)}{p_{1}} \frac{l_{1, b}^{\mathrm{fb}}}{l_{0}^{\mathrm{fb}}}=: \Delta_{A}$, then $\pi_{0}^{\mathcal{S} *}>\pi_{0}^{\mathcal{S} * *}$. Moreover, if $\psi l_{0}^{\mathrm{fb}}<l_{1, b}^{\mathrm{fb}}$ and thus $\Delta_{1}>\Delta_{A}$, then $\frac{\partial \psi}{\partial \Delta_{1}}<0$ and $\frac{\partial \pi_{0}^{\mathcal{S} *}}{\partial \psi}>0$ so that $\pi_{0}^{\mathcal{S} \mathcal{S} *}$ is decreasing in $\Delta_{1}$ while $\pi_{0}^{\mathcal{S R} *}$ is independent of $\Delta_{1}$. Therefore, we have $\pi_{0}^{\mathcal{S} *} \geq \pi_{0}^{\mathcal{S R} *}$ only if $\Delta_{1} \leq \Delta_{B}$ where $\Delta_{B}$ is implicitly defined by

$$
\left(1-p_{1}\right)\left(\phi_{1, b}^{\mathcal{S}}\left(\psi l_{0}^{\mathcal{S}}\right)-\phi_{1, b}^{\mathcal{R}}\left(l_{1, b}^{\mathcal{R}}\right)\right)=\phi_{0}^{\mathcal{S}}\left(l_{0}^{\mathrm{fb}}\right)-\phi_{0}^{\mathcal{S}}\left(l_{0}^{\mathcal{S}}\right)
$$


We can conclude

$$
\begin{array}{lll}
l_{0}^{\mathcal{S} *}=l_{0}^{\mathrm{fb}}, & \pi_{0}^{\mathcal{S} *}=p_{1} \phi_{1, g}^{\mathcal{S}}\left(l_{1, g}^{\mathrm{fb}}\right)+\left(1-p_{1}\right) \phi_{1, b}^{\mathcal{S}}\left(l_{1, b}^{\mathrm{fb}}\right)+\phi_{0}^{\mathcal{S}}\left(l_{0}^{\mathrm{fb}}\right) & \text { if } \Delta_{1} \leq \Delta_{A}, \\
l_{0}^{\mathcal{S} *}=l_{0}^{\mathcal{S}}, & \pi_{0}^{\mathcal{S} *}=p_{1} \phi_{1, g}^{\mathcal{S}}\left(l_{1, g}^{\mathrm{fb}}\right)+\left(1-p_{1}\right) \phi_{1, b}^{\mathcal{S}}\left(\psi l_{0}^{\mathcal{S}}\right)+\phi_{0}^{\mathcal{S}}\left(l_{0}^{\mathcal{S}}\right) & \text { if } \Delta_{1} \in\left(\Delta_{A}, \Delta_{B}\right], \\
l_{0}^{\mathcal{S} *}=l_{0}^{\mathrm{fb}}, & \pi_{0}^{\mathcal{S} *}=p_{1} \phi_{1, g}^{\mathcal{S}}\left(l_{1, g}^{\mathrm{fb}}\right)+\left(1-p_{1}\right) \phi_{1, b}^{\mathcal{R}}\left(l_{1, b}^{\mathcal{R}}\right)+\phi_{0}^{\mathcal{S}}\left(l_{0}^{\mathrm{fb}}\right) & \text { if } \Delta_{1} \in\left(\Delta_{B}, \widetilde{\Delta}_{1}\right] .
\end{array}
$$

Case II Suppose that $\Delta_{1}>\widetilde{\Delta}_{1}$ and thus $l_{1, b}^{\max } \leq 0$. Then, (A.7) implies $\frac{\partial l_{1, b}^{*}}{\partial l_{0}}=0$. Insertion of this in (A.8) yields $\pi_{0}^{\prime}=\phi_{0}^{\mathcal{S} \prime}\left(l_{0}\right)=0$ for $l_{0}=l_{0}^{\mathrm{fb}}$ and $\pi_{0}^{\prime \prime}<0$. Note that $l_{0}^{\mathrm{fb}} \leq l_{0}^{\mathrm{max}}$ only if $\Delta_{1} \leq \widetilde{\Delta}_{1}+\frac{\phi_{1, b}^{\mathcal{R}}\left(l_{1, b}^{\mathcal{R}}\right)}{p_{1} l_{0}^{\mathrm{fb}}}=: \Delta_{C}$. We obtain

$$
\begin{aligned}
& l_{0}^{\mathcal{S} *}=l_{0}^{\mathrm{fb}}, \quad \pi_{0}^{\mathcal{S} *}=p_{1} \phi_{1, g}^{\mathcal{S}}\left(l_{1, g}^{\mathrm{fb}}\right)+\left(1-p_{1}\right) \phi_{1, b}^{\mathcal{R}}\left(l_{1, b}^{\mathcal{R}}\right)+\phi_{0}^{\mathcal{S}}\left(l_{0}^{\mathrm{fb}}\right) \quad \text { if } \Delta_{1} \in\left(\widetilde{\Delta}_{1}, \Delta_{C}\right], \\
& l_{0}^{\mathcal{S} *}=l_{0}^{\max }, \quad \pi_{0}^{\mathcal{S} *}=p_{1} \phi_{1, g}^{\mathcal{S}}\left(l_{1, g}^{\mathrm{fb}}\right)+\left(1-p_{1}\right) \phi_{1, b}^{\mathcal{R}}\left(l_{1, b}^{\mathcal{R}}\right)+\phi_{0}^{\mathcal{S}}\left(l_{0}^{\max }\right) \quad \text { if } \Delta_{1}>\Delta_{C} .
\end{aligned}
$$

\section{Risky Mode}

For $m_{0}=\mathcal{R}$, insertion of $q_{1}=1-p_{1}$ into (8) yields $d_{1}=\frac{l_{0}+a_{0}}{p_{1}}$. Insertion of this into $\Omega_{1, s}:=v_{s} l_{0}+a_{0}-d_{1}$ for $s=g, b$ yields $\Omega_{1, g}=\frac{p_{1} v_{g}-1}{p_{1}} l_{0}-\frac{1-p_{1}}{p_{1}} a_{0}$ and $\Omega_{1, b}=\frac{p_{1} v_{b}-1}{p_{1}} l_{0}-\frac{1-p_{1}}{p_{1}} a_{0}$. Note that $\frac{\partial \Omega_{1, g}}{\partial a_{0}}<0$ and that (A.4) and (A.5) imply $\frac{\partial \pi_{1, g}^{*}}{\partial \Omega_{1, g}}>0$ while the restriction (9) on $m_{1, b}^{*}$ and (A.7) requires $\pi_{1, b}^{*}=0$. In conjunction with (7), we obtain $a_{0}^{*}=0$ and thus $\Omega_{1, g}=\frac{p_{1} v_{g}-1}{p_{1}} l_{0}>0$ and $\Omega_{1, b}=\frac{p_{1} v_{b}-1}{p_{1}} l_{0}$ so that (A.4) implies $\pi_{1, g}^{*}=\phi_{1, g}^{\mathcal{S}}\left(l_{1, g}^{\mathrm{fb}}\right)+\Omega_{1, g}$. Insertion of $\Omega_{1, g}, \pi_{1, g}^{*}$ and $\pi_{1, b}^{*}=0$ into (7) yields $\max _{l_{0}} \pi_{0}=p_{1} \phi_{1, g}^{\mathcal{S}}\left(l_{1, g}^{\mathrm{fb}}\right)+\phi_{0}^{\mathcal{R}}\left(l_{0}\right)$. Note that $\pi_{0}^{\prime}=\phi_{0}^{\mathcal{R} \prime}\left(l_{0}\right)=0$ for $l_{0}=l_{0}^{\mathcal{R}}<l_{0}^{\mathrm{fb}}$ where $l_{0}^{\mathcal{R}}: \phi_{0}^{\mathcal{R} \prime}\left(l_{0}^{\mathcal{R}}\right)=0$ and $\pi_{0}^{\prime \prime}<0$. We obtain

$$
l_{0}^{\mathcal{R} *}=l_{0}^{\mathcal{R}}, \quad \pi_{0}^{\mathcal{R} *}=p_{1} \phi_{1, g}^{\mathcal{S}}\left(l_{1, g}^{\mathrm{fb}}\right)+\phi_{0}^{\mathcal{R}}\left(l_{0}^{\mathcal{R}}\right) .
$$

\section{Optimal Behavior}

We now compare $\pi_{0}^{\mathcal{S} *}$ and $\pi_{0}^{\mathcal{R} *}$ with the expected payoff $\pi_{0}^{\mathcal{F} *}=0$ of the failure mode.

- If $\Delta_{1} \leq \Delta_{C}$, then (A.10), (A.11) and (A.12) imply $\pi_{0}^{\mathcal{S} *}>\pi_{0}^{\mathcal{R} *}>0$.

- If $\Delta_{1}>\Delta_{C}$, then (A.11) and (A.12) imply that $\pi_{0}^{\mathcal{S} *}$ is decreasing in $\Delta_{1}$ (since $l_{0}^{\max }$ is decreasing in $\Delta_{1}$ ) while $\pi_{0}^{\mathcal{R} *}>0$ is independent of $\Delta_{1}$. Therefore, we have $\pi_{0}^{\mathcal{S} *} \geq$ 
$\pi_{0}^{\mathcal{R} *}>0$ only if $\Delta \leq \Delta_{D}$ where $\Delta_{D}$ is defined by

$$
\left(1-p_{1}\right) \phi_{1, b}^{\mathcal{R}}\left(l_{1, b}^{\mathcal{R}}\right)=\phi_{0}^{\mathcal{R}}\left(l_{0}^{\mathcal{R}}\right)-\phi_{0}^{\mathcal{S}}\left(\frac{\phi_{1, b}^{\mathcal{R}}\left(l_{1, b}^{\mathcal{R}}\right)}{p_{1}\left(\Delta_{D}-\widetilde{\Delta}_{1}\right)}\right)
$$

In conjunction with (A.10), (A.11) and (A.12), these two cases immediately lead to (11). 